\title{
On some generalized equations with metrically $C$-increasing mappings: solvability and error bounds with applications to optimization
}

\author{
A. Uderzo $^{\mathrm{a}}$ \\ ${ }^{a}$ Department of Mathematics and Applications, Università di Milano-Bicocca, Milano, Italy
}

\author{
ARTICLE HISTORY \\ Compiled December 6, 2018
}

\begin{abstract}
Generalized equations are problems emerging in contexts of modern variational analysis as an adequate formalism to treat such issues as constraint systems, optimality and equilibrium conditions, variational inequalities, differential inclusions. The present paper contains a study on solvability and error bounds for generalized equations of the form $F(x) \subseteq C$, where $F$ is a given set-valued mapping and $C$ is a closed, convex cone. A property called metric $C$-increase, matching the metric behaviour of $F$ with the partial order associated with $C$, is singled out, which ensures solution existence and error bound estimates in terms of problem data. Applications to the exact penalization of optimization problems with constraint systems, defined by the above class of generalized equations, and to the existence of ideal efficient solutions in vector optimization are proposed.
\end{abstract}

\section{KEYWORDS}

Generalized equations; metrically $C$-increasing mapping; openness at a linear rate; solvability; error bound; vector optimization; exact penalization

\section{Introduction}

A feature distinguishing modern variational analysis is the set-oriented approach to traditional subjects of study. The language in which many fundamental results are formulated as well as the comprehensive apparatus of notions and constructions lying at the core of its theory should make this statement evident. Reasons for such a feature can be found in developments of the last half-century in such areas as optimization and optimal control. There, with the aim of addressing a broad spectrum of extremum problems arising in applications, the need to treat situations falling out from the classical analysis emerged urgently. As a result, traditional categories of the mathematical thought such as vectors, equations and functions now share the center of the stage with new entries, like sets, inequalities, and multifunctions, considered per se worthwhile of a dedicated calculus and investigations, not less than the former ones. Generalized equations, whose study was initiated by S.M. Robinson (see [1,2]), are problems that seem to be paradigmatic of this trend. Involving multifunctions and sets in their very structure, they are powerful enough to subsume standard (equality/inequality) constraint systems as well as other relations and/or geometric constraints, various optimality and equilibrium conditions (often expressed in form

CONTACT A. Uderzo Email: amos.uderzo@unimib.it 
of inclusion or inequalities), variational inequalities and complementarity problems. So, the study of all the above topics received an effective impulse from advances in the theory of generalized equations. Among the central questions in this branch of variational analysis are solvability and solution stability, error bound estimates, as well as the sensitivity analysis of solution sets, often leading to implicit multifunction theorems in the case of parameterized generalized equations (see, among others, [2], [3, Ch. 5], [4, Ch. 5], [5, Ch. 2 and 4], [6, Ch. 4.4]).

In this paper, the main subjects of study are solvability and error bounds for the following type of generalized equations

$$
\text { find } x \in X \text { such that } F(x) \subseteq C \text {, }
$$

where $F: X \rightrightarrows \mathbb{Y}$ is a given set-valued mapping and $C \subseteq \mathbb{Y}$ is a nonempty closed and convex subset of a normed space. This means the study of conditions on the problem data $F$ and $C$, under which the related solution set, i.e.

$$
\mathcal{S o l}(F, C)=\{x \in X: F(x) \subseteq C\},
$$

is nonempty and certain inequalities measuring the distance from $\operatorname{Sol}(F, C)$ hold true. Whereas a well-developed theory exists for generalized equations of the form

$$
\mathbf{0} \in f(x)+G(x)
$$

with 0 being the null element of $\mathbb{Y}, f: X \longrightarrow \mathbb{Y}$ and $G: X \rightrightarrows \mathbb{Y}$ being given data, the case of generalized equations (GE) attracted so far a minor interest. Yet, a format like (GE) emerges in various contexts of optimization theory, as illustrated by the next example. Besides, in the particular case in which $F$ in (GE) is a single-valued mapping and it is $G \equiv-C$ in (1), then (GE) and (11) collapse to the same problem.

Example 1.1. (i) Let $f: X \longrightarrow \mathbb{Y}$ be a function taking values in a vector space $\mathbb{Y}$ partially ordered by its (positive) cone $C$ and let $R \subseteq X$ be a nonempty set. Recall that $\bar{x} \in R$ is said to be an ideally $C$-efficient solution for the related vector optimization problem

(VOP) $\quad C$ - $\min f(x) \quad$ subject to $\quad x \in R$,

provided that

$$
f(R) \subseteq f(\bar{x})+C
$$

Thus, introducing the set-valued mapping $F: X \rightrightarrows \mathbb{Y}$ defined by $F(x)=f(R)-f(x)$, one gets that the set of all ideally $C$-efficient solutions coincides with $\mathcal{S}$ ol $(F, C)$. It is worth recalling that any ideal $C$-efficient solution is, in particular, also $C$-efficient (see [7]).

(ii) Essentially, semi-infinite programs are optimization problems, whose variables lie in a finite-dimensional space, that are subject to infinitely many constrains (see [8]). Using $T$ as an (infinite) index set, a semi-infinite programming problem can be formalized as follows

$$
\min _{x \in S} \varphi(x) \quad \text { subject to } g(t, x) \leq 0, \forall t \in T
$$


where $S \in \mathbb{R}^{n}$ represents a geometric constraint, $\varphi: \mathbb{R}^{n} \longrightarrow \mathbb{R}$ and $g: T \times \mathbb{R}^{n} \longrightarrow \mathbb{R}$ are given functions. It is readily seen that, setting $F(x)=\{g(t, x): t \in T\}=g(T, x)$ and $C=(-\infty, 0]$, the feasible region of such kind of problems becomes $\operatorname{Sol}(F, C)$.

(iii) Consider a standard constrained convex optimization problem, namely

$$
\min _{x \in S} \varphi(x) \quad \text { subject to } \quad x \in R
$$

where $\varphi: \mathbb{X} \longrightarrow \mathbb{R}$ is a convex smooth functional defined on a normed space $\mathbb{X}$ and $R \subset \mathbb{X}$ is a nonempty, closed and convex set. It is well known that the (global) optimality of an element $\bar{x} \in R$ is characterized by the variational inequality

$$
\langle\nabla \varphi(\bar{x}), x-\bar{x}\rangle \geq 0, \quad \forall x \in R
$$

where $\nabla \varphi(\bar{x})$ denotes the Gâteaux derivative of $\varphi$ at $\bar{x}$ (see, for instance, [9, Proposition 5.1.1]). Thus, if setting $F(x)=\langle\nabla \varphi(x), R-x\rangle$ and $C=[0,+\infty), \mathcal{S} o l(F, C)$ coincides with the set of all global solutions to the above optimization problem.

(iv) In mathematical economics, a production process consists of the transformation of production factors (scarce resources), or inputs, into products (goods, services), or outputs. A production technology is a description of the relationships between inputs and outputs (see, for instance, $[10,11]$ ). Such a description may be quantitatively formalized by a set-valued mapping $F: \mathbb{R}^{n} \rightrightarrows \mathbb{R}^{m}$ associating with each output $x \in \mathbb{R}^{n}$ the set consisting of all inputs $y \in \mathbb{R}^{m}$ needed to produce $x$ (it is reasonable to assume that the same output can be obtained by means of different combinations of inputs). Given a cone $C$, any condition like (GE) can be interpreted as a constraint on the production technology, corresponding to specific requirements on the input employment.

Clearly, the study of such issues as solvability and error bounds for (GE) can be performed through several approaches. The present paper proposes an approach, which relies on a property for set-valued mappings here introduced, called metric $C$-increase. This notion captures a behaviour of set-valued mappings that combines the partial order induced by the cone $C$ with metric variations of the given mappings. Roughly speaking, such property works as a generalization to a set-valued setting of a decrease principle. In synergy with the Ekeland variational principle, metric $C$-increase turns out to guarantee solution existence results for (GE) as well as quantitative estimates of the distance from the solution set. It is worth mentioning that a different approach to the study of error bound for the above (GE ) has been considered in [12]. According to such an approach, which rests upon techniques of convex analysis, the set-valued mapping $F$ is required to satisfy generalized convexity assumptions, the cone $C$ is required to have nonempty interior and the solution set to be nonempty. None of these assumptions will be made according the approach presented here.

The arrangement of the contents of the paper in the subsequent sections is as follows. In Section 2 some technical preliminaries, adequated to the analysis to be carried out, are recalled. Essentially, they deal with translations of set enlargements, with 'additivelike' properties of the excess function and with semicontinuity properties of set-valued mappings. In Section 3 the notion of metric $C$-increase for set-valued mappings is introduced, both in its global and local form, along with the related exact bounds. Some examples are provided and some connections with well-known properties in variational analysis are explored. Section 4 is devoted to exposing the main results of the paper, which refer to solvability and error bounds for generalized equations of the 
form (GE). In Section 5 some applications to optimization are discussed: the first one refers to the existence of ideal efficient solutions to vector optimization problems; a further application concerns the exact penalization of general optimization problems, whose constraint systems are defined by generalized equations of the form (GE).

\section{Technical preliminaries}

The notation in use throughout the paper is standard. $\mathbb{N}$ and $\mathbb{R}$ denote the natural and the real number set, respectively. $\mathbb{R}_{+}^{m}$ denotes the nonnegative orthant in the space $\mathbb{R}^{m}$. Whenever $x$ is an element of a metric space $(X, d)$ and $r$ is a positive real, $\mathrm{B}(x, r)=\{z \in X: d(z, x) \leq r\}$ denotes the closed ball with center $x$ and radius $r$. Given a subset $S$ of a metric space, by int $S$ its topological interior is denoted. By dist $(x, S)=\inf _{z \in S} d(z, x)$ the distance of $x$ from a subset $S \subseteq X$ is denoted, with the convention that dist $(x, \varnothing)=+\infty$. The $r$-enlargement of a set $S \subseteq X$ is indicated by $\mathrm{B}(S, r)=\{x \in X: \operatorname{dist}(x, S) \leq r\}$. Given a pair of subsets $S_{1}, S_{2} \subseteq X$, the symbol $\operatorname{exc}\left(S_{1}, S_{2}\right)=\sup _{s \in S_{1}} \operatorname{dist}\left(s, S_{2}\right)$ denotes the excess of $S_{1}$ over $S_{2}$. The null vector in a normed space is indicated by $\mathbf{0}$. Thus, $\mathbb{B}=\mathrm{B}(\mathbf{0}, 1)$ and $\mathbb{S}=\{x \in \mathbb{B}:\|x\|=1\}$ stand for the unit ball and the unit sphere in a normed space, respectively. Whenever $F: X \rightrightarrows \mathbb{Y}$ is a set-valued mapping, gph $F$ and $\operatorname{dom} F$ denote the graph and the domain of $F$, respectively. All the set-valued mappings appearing in the paper will be supposed to take closed values. Throughout the text, the acronyms l.s.c. and u.s.c. stand for lower semicontinuous and upper semicontinuous, respectively. Further more specific notations will be introduced contextually to their use.

Throughout the current section, $(\mathbb{Y},\|\cdot\|)$ denotes a real normed space. For the reader's convenience, in the next remark some basic facts concerning the interaction between enlargements of a set, translations and the excess function, which will be employed in the sequel, are collected. Their proof can be derived as a direct consequence of the definition of the involved objects.

Remark 1. Let $S \subset \mathbb{Y}$ be a nonempty subset and let $r>0$.

(i) It holds

$$
\bigcup_{y \in S} \mathrm{~B}(y, r) \subseteq \mathrm{B}(S, r) \subseteq \bigcap_{\epsilon>0} \bigcup_{y \in S} \mathrm{~B}(y, r+\epsilon)
$$

If, in particular, $S$ is a closed subset of a finite-dimensional Euclidean space, then

$$
\bigcup_{y \in S} \mathrm{~B}(y, r)=\mathrm{B}(S, r)
$$

(ii) Let $y \in \mathbb{Y}$. Then, it holds

$$
\mathrm{B}(y, r)+C \subseteq \mathrm{B}(y+C, r) \subseteq \bigcap_{\epsilon>0}(\mathrm{~B}(y, r+\epsilon)+C)
$$

If, in particular, $C$ is a closed subset of a finite-dimensional Euclidean space, then

$$
\mathrm{B}(y+C, r)=\mathrm{B}(y, r)+C .
$$


(iii) Let $S$ be a nonempty subset. It holds

$$
\mathrm{B}(S, r)+C \subseteq \mathrm{B}(S+C, r) \subseteq \bigcap_{\epsilon>0} \mathrm{~B}(S+C, r+\epsilon)
$$

If, in particular, $S$ and $C$ are nonempty subsets of a finite-dimensional Euclidean space such that $S+C$ is closed, then

$$
\mathrm{B}(S, r)+C=\mathrm{B}(S+C, r) .
$$

(iv) Let $C \subset \mathbb{Y}$ be a closed, convex cone. Then, it results in

$$
\operatorname{exc}(S, C)=\operatorname{exc}(S+C, C)
$$

(v) Let $S_{1}$ and $S_{2}$ be nonempty subsets of $\mathbb{Y}$ and let $r_{1}, r_{2}>0$. Then,

$$
\mathrm{B}\left(S_{1}, r_{1}\right)+\mathrm{B}\left(S_{2}, r_{2}\right) \subseteq \bigcap_{\epsilon>0} \mathrm{~B}\left(S_{1}+S_{2}, r_{1}+r_{2}+\epsilon\right) .
$$

If, in particular, $\mathbb{Y}$ is a finite-dimensional Euclidean space, then

$$
\mathrm{B}\left(S_{1}, r_{1}\right)+\mathrm{B}\left(S_{2}, r_{2}\right) \subseteq \mathrm{B}\left(S_{1}+S_{2}, r_{1}+r_{2}\right) .
$$

(vi) If $C \subset \mathbb{Y}$ is a closed, convex cone, with $\bar{c} \in C$ and $y \in \mathbb{Y} \backslash C$, and $\alpha>0$, then

$$
\operatorname{dist}(y+\alpha(y-\bar{c}), C) \geq(1+\alpha) \operatorname{dist}(y, C) \text {. }
$$

Indeed, from

$$
\operatorname{dist}(y+\alpha(y-\bar{c}), C)=\inf _{c \in C}\|(1+\alpha) y-\alpha \bar{c}-c\|,
$$

as it is $\alpha \bar{c}+C \subseteq C$, it follows

$$
\begin{aligned}
\inf _{c \in C}\|(1+\alpha) y-\alpha \bar{c}-c\| & \geq \inf _{c \in C}\|(1+\alpha) y-c\| \\
& =\inf _{c \in C}\|(1+\alpha) y-c(1+\alpha)\|=(1+\alpha) \operatorname{dist}(y, C) .
\end{aligned}
$$

On the basis of some of the facts recalled above, the following technical lemma, expressing a sort of additivity of the excess with respect to the radius of balls, can be derived.

Lemma 2.1. Let $C \subset \mathbb{Y}$ be a closed, convex cone and let $y \in \mathbb{Y} \backslash C$. Then, for any $r>0$ it holds

$$
\operatorname{exc}(\mathrm{B}(y, r), C)=\operatorname{dist}(y, C)+r .
$$

Proof. Let us show first that $\operatorname{exc}(\mathrm{B}(y, r), C) \leq \operatorname{dist}(y, C)+r$. Let $v \in r \mathbb{B}$. From

$$
\inf _{c \in C}\|y+v-c\| \leq \inf _{c \in C}\|y-c\|+\|v\|
$$


one readily obtains

$$
\operatorname{exc}(\mathrm{B}(y, r), C)=\sup _{v \in r \mathbb{B}} \inf _{c \in C}\|y+v-c\| \leq \sup _{v \in r \mathbb{B}}\left[\inf _{c \in C}\|y-c\|+\|v\|\right]=\operatorname{dist}(y, C)+r
$$

To prove the converse inequality, fix an arbitrary $\epsilon>0$. Correspondingly, there exists $c_{\epsilon} \in C$ such that $\left\|y-c_{\epsilon}\right\|<(1+\epsilon) \operatorname{dist}(y, C)$ (remember that $\left.\operatorname{dist}(y, C)>0\right)$. Thus, since $y \neq c_{\epsilon}$, by recalling inequality (2) with $\alpha=\left\|y-c_{\epsilon}\right\|^{-1} r$, one obtains

$$
\begin{aligned}
\operatorname{exc}(\mathrm{B}(y, r), C) & =\sup _{v \in r \mathbb{B}} \inf _{c \in C}\|y+v-c\| \geq \inf _{c \in C}\left\|y+\frac{y-c_{\epsilon}}{\left\|y-c_{\epsilon}\right\|} r-c\right\| \\
& \geq\left(1+\frac{r}{\left\|y-c_{\epsilon}\right\|}\right) \operatorname{dist}(y, C) \geq \operatorname{dist}(y, C)+\frac{r}{1+\epsilon} .
\end{aligned}
$$

By letting $\epsilon \rightarrow 0^{+}$, one achieves the inequality

$$
\operatorname{exc}(\mathrm{B}(y, r), C) \geq \operatorname{dist}(y, C)+r
$$

thereby completing the proof.

The next lemma, which will be employed in the proof of the main result of the paper, generalizes the previous relation to enlargements of closed sets.

Lemma 2.2. Let $C \subseteq \mathbb{Y}$ be a closed, convex cone and let $S \subseteq \mathbb{Y}$ be a nonempty subset such that $\operatorname{exc}(S, C)>0$. For any $r>0$ it holds

$$
\operatorname{exc}(\mathrm{B}(S, r), C)=\operatorname{exc}(S, C)+r
$$

Proof. Observe first that

$$
\operatorname{exc}(S, C)=\sup _{y \in S} \operatorname{dist}(y, C)=\sup _{y \in S \backslash C} \operatorname{dist}(y, C)=\operatorname{exc}(S \backslash C, C) .
$$

Thus, according to Lemma 2.1, in particular one has that for every $y \in S \backslash C$ it is $\operatorname{dist}(y, C)=\operatorname{exc}(\mathrm{B}(y, r), C)-r$. It follows

$$
\begin{aligned}
\operatorname{exc}(S, C) & =\sup _{y \in S \backslash C} \operatorname{dist}(y, C)=\sup _{y \in S \backslash C} \operatorname{exc}(\mathrm{B}(y, r), C)-r \\
& =\sup _{y \in S \backslash C} \sup _{v \in \mathrm{B}(y, r)} \operatorname{dist}(v, C)-r \leq \sup _{v \in \mathrm{B}(S, r)} \operatorname{dist}(v, C)-r \\
& =\operatorname{exc}(\mathrm{B}(S, r), C)-r
\end{aligned}
$$

where the last inequality holds because, as observed in Remark 1(i), it holds

$$
\bigcup_{y \in S \backslash C} \mathrm{~B}(y, r) \subseteq \bigcup_{y \in S} \mathrm{~B}(y, r) \subseteq \mathrm{B}(S, r) .
$$

Now, in order to establish the converse inequality, fix an arbitrary $\epsilon>0$. Since as noticed in Remark 1(i) it is $\mathrm{B}(S, r) \subseteq \cup_{y \in S} \mathrm{~B}(y, r+\epsilon)$, by applying Lemma 2.1, one 
finds

$$
\begin{aligned}
\operatorname{exc}(\mathrm{B}(S, r), C) & \leq \operatorname{exc}\left(\cup_{y \in S} \mathrm{~B}(y, r+\epsilon), C\right)=\sup _{y \in S} \operatorname{exc}(\mathrm{B}(y, r+\epsilon), C) \\
& =\sup _{y \in S} \operatorname{dist}(y, C)+r+\epsilon=\operatorname{exc}(S, C)+r+\epsilon .
\end{aligned}
$$

The arbitrariness of $\epsilon$ completes the proof.

Let us point out below a consequence of Lemma 2.2 that will be useful in the sequel.

Remark 2. Given a closed, convex cone $S \subset \mathbb{Y}$, a nonempty set $S \subseteq \mathbb{Y}$ and constants $r>0$ and $a>1$, it holds

$$
\mathrm{B}(S, a r) \nsubseteq \mathrm{B}(S+C, r) .
$$

Indeed, if assuming that $\mathrm{B}(S, a r) \subseteq \mathrm{B}(S+C, r)$, then according to Lemma 2.2 and Remark 1(iv), one would obtain

$$
\begin{aligned}
\operatorname{exc}(S, C)+a r & =\operatorname{exc}(\mathrm{B}(S, a r), C) \leq \operatorname{exc}(\mathrm{B}(S+C, r), C) \\
& =\operatorname{exc}(S+C, C)+r=\operatorname{exc}(S, C)+r
\end{aligned}
$$

which leads to the evident contradiction $a r \leq r$.

In view of the formulation of the next ancillary result, recall that a set-valued mapping $F: X \rightrightarrows \mathbb{Y}$ defined on a metric space is said to be l.s.c. at $\bar{x}$ if for every open set $O \subseteq \mathbb{Y}$ such that $F(\bar{x}) \cap O \neq \varnothing$ there exists $\delta_{O}>0$ such that

$$
F(x) \cap O \neq \varnothing, \quad \forall x \in \mathrm{B}\left(\bar{x}, \delta_{O}\right) .
$$

Given a closed convex cone $C \subset \mathbb{Y}, F$ is said to be Hausdorff $C$-u.s.c. at $\bar{x}$ if for every $\epsilon>0$ there exists $\delta_{\epsilon}>0$ such that

$$
F(x) \subseteq \mathrm{B}(F(\bar{x})+C, \epsilon), \quad \forall x \in \mathrm{B}\left(\bar{x}, \delta_{\epsilon}\right) .
$$

The next lemma links the above semicontinuity properties of a set-valued mapping $F$ with the semicontinuity properties of the function $\phi: X \longrightarrow[0,+\infty)$, defined by

$$
\phi(x)=\operatorname{exc}(F(x), C),
$$

which is possible to associate with $F$ and $C$. Not surprisingly, such a function will play a crucial role in the main achievements in this paper.

Lemma 2.3. Let $F: X \rightrightarrows \mathbb{Y}$ be a set-valued mapping defined on a metric space $(X, d)$ and taking values on a normed space $(\mathbb{Y},\|\cdot\|)$, and let $C \subset \mathbb{Y}$ be a closed, convex cone.

(i) If $F$ is l.s.c. at $\bar{x}$, then the function $\phi$ is l.s.c. at $\bar{x}$.

(ii) If $F$ is Hausdorff C-u.s.c. at $\bar{x}$, then the function $\phi$ is u.s.c. at $\bar{x}$.

Proof. (i) Fix $\bar{x} \in X$. If $F(\bar{x})=\varnothing$, then $\phi(\bar{x})=\sup _{y \in \varnothing} \operatorname{dist}(y, C)=-\infty$. Thus, for any sequence $\left(x_{n}\right)_{n \in \mathbb{N}}$ in $X$, with $x_{n} \longrightarrow \bar{x}$ as $n \rightarrow \infty$, the inequality

$$
\liminf _{n \rightarrow \infty} \phi\left(x_{n}\right) \geq-\infty=\phi(\bar{x})
$$


is trivially fulfilled.

If $F(\bar{x}) \neq \varnothing$ and $F(\bar{x}) \subseteq C$, then $\phi(\bar{x})=0$, so one finds

$$
\liminf _{n \rightarrow \infty} \phi\left(x_{n}\right) \geq 0=\phi(\bar{x})
$$

Now, assume that $F(\bar{x}) \not \subset C$, so that

$$
\phi(\bar{x})=\operatorname{exc}(F(\bar{x}), C)=\sup _{y \in F(\bar{x})} \operatorname{dist}(y, C)>0 .
$$

Fix an arbitrary $\epsilon \in(0, \phi(\bar{x}))$. Then, corresponding to $\epsilon$, there exists $y_{\epsilon} \in F(\bar{x})$ such that

$$
\operatorname{dist}\left(y_{\epsilon}, C\right)>\phi(\bar{x})-\frac{\epsilon}{2} \text {. }
$$

Since $F$ is l.s.c. at $\bar{x}$, there exists $r_{\epsilon}>0$ such that

$$
F(x) \cap \operatorname{int} \mathrm{B}\left(y_{\epsilon}, \epsilon / 2\right) \neq \varnothing, \quad \forall x \in \mathrm{B}\left(\bar{x}, r_{\epsilon}\right) .
$$

Thus, if $\left(x_{n}\right)_{n \in \mathbb{N}}$ is any sequence in $X$, with $x_{n} \longrightarrow \bar{x}$, then for some $n_{*} \in \mathbb{N}$ one gets the existence of $y_{n} \in F\left(x_{n}\right) \cap \operatorname{int} \mathrm{B}\left(y_{\epsilon}, \epsilon / 2\right)$ for every $n \geq n_{*}$. On the account of inequality (4), one obtains

$$
\phi\left(x_{n}\right) \geq \operatorname{dist}\left(y_{n}, C\right) \geq \operatorname{dist}\left(y_{\epsilon}, C\right)-d\left(y_{n}, y_{\epsilon}\right)>\phi(\bar{x})-\epsilon, \quad \forall n \in \mathbb{N}, n \geq n_{*},
$$

wherefrom it follows

$$
\liminf _{n \rightarrow \infty} \phi\left(x_{n}\right) \geq \phi(\bar{x})-\epsilon .
$$

By arbitrariness of $\epsilon$, the thesis follows.

(ii) Let $\left(x_{n}\right)_{n \in \mathbb{N}}$ be any sequence in $X$ converging to $\bar{x}$ as $n \rightarrow \infty$. Now, it is to be proved that

$$
\limsup _{n \rightarrow \infty} \phi\left(x_{n}\right) \leq \phi(\bar{x})
$$

If $F(\bar{x})=\varnothing$, then $\phi(\bar{x})=\sup _{y \in \varnothing} \operatorname{dist}(y, C)=+\infty$, so the inequality to be proved trivially holds. Suppose that $F(\bar{x}) \neq \varnothing$ and fix $\epsilon>0$. Since $F$ is Hausdorff $C$-u.s.c. at $\bar{x}$, corresponding to $\epsilon$ there exists $\delta_{\epsilon}>0$ such that

$$
F(x) \subseteq \mathrm{B}(F(\bar{x})+C, \epsilon), \quad \forall x \in \mathrm{B}\left(\bar{x}, \delta_{\epsilon}\right) .
$$

As $x_{n} \longrightarrow \bar{x}$, there exists $n_{*} \in \mathbb{N}$ such that $x_{n} \in \mathrm{B}\left(\bar{x}, \delta_{\epsilon}\right)$, for every $n \in \mathbb{N}$, with $n \geq n_{*}$. Consequently, in force of Lemma 2.2 and Remark 1(iv), one obtains

$$
\begin{aligned}
\phi\left(x_{n}\right) & =\operatorname{exc}\left(F\left(x_{n}\right), C\right) \leq \operatorname{exc}(\mathrm{B}(F(\bar{x})+C, \epsilon), C)=\operatorname{exc}(F(\bar{x})+C, C)+\epsilon \\
& =\operatorname{exc}(F(\bar{x}), C)+\epsilon=\phi(\bar{x})+\epsilon, \quad \forall n \geq n_{*} .
\end{aligned}
$$


From the last inequality, it follows

$$
\limsup _{n \rightarrow \infty} \phi\left(x_{n}\right) \leq \phi(\bar{x})+\epsilon
$$

which, by arbitrariness of $\epsilon$, completes the proof.

\section{Metrically $C$-increasing mappings}

Throughout the current section, by $F: X \rightrightarrows \mathbb{Y}$ a set-valued mapping is denoted which is defined on a metric space $(X, d)$ and takes values in a real normed space $(\mathbb{Y},\|\cdot\|)$, partially ordered by a closed, convex cone $C \subset \mathbb{Y}$. This means that, associated in a standard way with the cone $C$, a partial order relation $\leq_{C} \subset \mathbb{Y} \times \mathbb{Y}$ is defined as follows

$$
y_{1} \leq_{C} y_{2} \quad \text { iff } \quad y_{2}-y_{1} \in C .
$$

The next definition introduces a property for set-valued mappings which plays a key role in establishing a solvability result for generalized equation of the form (GE), along with related error bound estimates.

Definition 3.1. (i) A mapping $F: X \rightrightarrows \mathbb{Y}$ is said to be metrically $C$-increasing on $X$ if there exists a constant $a>1$ such that

$$
\forall x \in X, \forall r>0, \exists u \in \mathrm{B}(x, r): \mathrm{B}(F(u), a r) \subseteq \mathrm{B}(F(x)+C, r) .
$$

The quantity

$$
\operatorname{inc}(F ; X)=\sup \{a>1: \text { inclusion (5) holds }\}
$$

is called exact bound of metric $C$-increase of $F$ on $X$.

(ii) A mapping $F: X \rightrightarrows \mathbb{Y}$ is said to be metrically $C$-increasing around $\bar{x} \in \operatorname{dom} F$ if there exist $\delta>0$ and $a>1$ such that

$$
\forall x \in \mathrm{B}(\bar{x}, \delta), \forall r \in(0, \delta), \exists u \in \mathrm{B}(x, r): \mathrm{B}(F(u), a r) \subseteq \mathrm{B}(F(x)+C, r) .
$$

The quantity

$$
\operatorname{inc}(F ; \bar{x})=\sup \{a>1: \exists \delta>0 \text { such that inclusion (6) holds }\}
$$

is called exact bound of metric $C$-increase of $F$ around $\bar{x}$.

The meaning of the property introduced above should appear from inclusion (5). Loosely speaking, it postulates that the values taken by $F$ near a given point 'move', among other directions, towards the cone $C$ in a manner which is proportional, with a certain rate, to the distance from the given point. The exact bound of metric $C$ increase provides a quantitative estimate of such a behaviour. It should be noticed that this notion is not connected with that of monotonicity, inasmuch as on the space $X$ any partial order or vector structure is lacking. The term 'metric $C$-increase' indeed refers to the matching of a metric behaviour with the partial order induced by $C$ on the range space $\mathbb{Y}$. 
The following examples provide concrete classes of mappings fulfilling the property under study and, at the same time, hint connections with another property widely investigated in variational analysis. In what follows, $\mathcal{L}(\mathbb{X}, \mathbb{Y})$ denotes the (Banach) space of all linear bounded operators between the Banach spaces $\mathbb{X}$ and $\mathbb{Y}$, endowed with the operator norm. In particular, $\mathbb{X}^{*}=\mathcal{L}(\mathbb{X}, \mathbb{R})$.

Example 3.2 (Regular linear mappings). Recall that a linear bounded operator $\Lambda$ : $\mathbb{X} \longrightarrow \mathbb{Y}$ between Banach spaces is said to be open at a linear rate if there exists a constant $\alpha>0$ such that

$$
\Lambda \mathbb{B} \supseteq \alpha \mathbb{B} .
$$

It is well known that the celebrated Banach-Schauder theorem provides a characterization for such a property: $\Lambda$ is open at a linear rate iff $\Lambda$ is onto, i.e. $\Lambda \mathbb{X}=\mathbb{Y}$. The quantity

$$
\operatorname{sur} \Lambda=\sup \{\alpha>0: \Lambda \mathbb{B} \supseteq \alpha \mathbb{B}\}
$$

is called the exact openness bound of $\Lambda$. Roughly speaking, it gives a quantitative description of the openness at a linear rate. Such a constant can be estimated in primal and in dual terms as follows: it holds

$$
\operatorname{sur} \Lambda=\frac{1}{\left\|\Lambda^{-1}\right\|^{-}}
$$

where $\Lambda^{-1}: \mathbb{Y} \rightrightarrows \mathbb{X}$ is the inverse (in general) set-valued mapping to $\Lambda$, which is always positively homogeneous and convex, while $\|\cdot\|^{-}$denotes the inner norm of a positively homogeneous mapping, namely if $H: \mathbb{Y} \rightrightarrows \mathbb{X}$, it is

$$
\|H\|^{-}=\sup _{y \in \mathbb{B}} \inf _{x \in H(y)}\|x\|
$$

besides, it holds

$$
\operatorname{sur} \Lambda=\inf _{\left\|u^{*}\right\|=1}\left\|\Lambda^{\top} u^{*}\right\|=\operatorname{dist}\left(\mathbf{0}, \Lambda^{\top} \mathbb{S}\right)
$$

where $\Lambda^{\top} \in \mathcal{L}\left(\mathbb{Y}^{*}, \mathbb{X}^{*}\right)$ denotes the adjoint operator to $\Lambda$ (see, for instance, [6, Corollary $1.58]$ ). Now, let $\mathbb{Y}=\mathbb{R}^{m}$ be equipped with its classical Euclidean space structure, let $C=\mathbb{R}_{+}^{m}$ and $m \geq 2$. In this setting, consider a linear bounded operator $\Lambda: \mathbb{X} \longrightarrow \mathbb{R}^{m}$ such that

$$
\operatorname{sur} \Lambda>m \text {. }
$$

This means that

$$
\Lambda \mathbb{B} \supseteq m \mathbb{B} .
$$


Denote by $\mathcal{B}=\left\{e^{1}, \ldots, e^{m}\right\}$ the canonical base of $\mathbb{R}^{m}$ and define

$$
e=\sqrt{m} \sum_{i=1}^{m} e^{i}
$$

It is clear that $e \in m \mathbb{B}$. Then, because of inclusion (8), there exists $u \in \mathbb{B}$ such that $\Lambda u=e$. On the other hand, notice that $\mathrm{B}(e, \sqrt{m}) \subseteq \mathbb{R}_{+}^{m}$. Indeed, if $y \in \mathrm{B}(e, \sqrt{m})$, it is $\|y-e\| \leq \sqrt{m}$, and hence

$$
\left|y_{i}-\sqrt{m}\right| \leq \sqrt{m}, \quad \forall i=1, \ldots, m
$$

wherefrom one gets $y_{i} \geq 0$, for every $i=1, \ldots, m$. Thus, it is possible to deduce the existence of $u \in \mathbb{B}$ such that

$$
\mathrm{B}(\Lambda u, \sqrt{m})=\mathrm{B}(e, \sqrt{m}) \subseteq \mathbb{R}_{+}^{m} \subseteq \mathbb{B}+\mathbb{R}_{+}^{m}=\mathrm{B}\left(\Lambda \mathbf{0}+\mathbb{R}_{+}^{m}, 1\right)
$$

where the last equality follows on the account of Remark 1(iii). By exploiting the linearity of $\Lambda$, from the last inclusion one readily obtains that for every $r>0$ there exists $u \in r \mathbb{B}$ such that

$$
\mathrm{B}(\Lambda u, r \sqrt{m}) \subseteq \mathrm{B}\left(\Lambda \mathbf{0}+\mathbb{R}_{+}^{m}, r\right)
$$

and for every $x \in \mathbb{X}$ and for every $r>0$ there exists $u \in \mathrm{B}(x, r)$ such that

$$
\mathrm{B}(\Lambda u, r \sqrt{m}) \subseteq \mathrm{B}\left(\Lambda x+\mathbb{R}_{+}^{m}, r\right)
$$

Since $\sqrt{m}>1$, the above reasoning shows that any linear mapping $\Lambda \in \mathcal{L}\left(\mathbb{X}, \mathbb{R}^{m}\right)$, with the property that sur $\Lambda>m$, turns out to be metrically $\mathbb{R}_{+}^{m}$-increasing on $\mathbb{X}$ with exact increase bound $\operatorname{inc}(\Lambda ; \mathbb{X}) \geq \sqrt{m}$.

Example 3.3. Let $\Lambda \in \mathcal{L}\left(\mathbb{X}, \mathbb{R}^{m}\right)$ and let $C \subset \mathbb{R}^{m}$ be a closed, convex cone. Assume that inc $(\Lambda ; \mathbb{X})>a$. Then, the set-valued mapping $L: \mathbb{X} \rightrightarrows \mathbb{R}^{m}$, defined by

$$
L(x)=\Lambda x+C
$$

is metrically $C$-increasing and $\operatorname{inc}(L ; \mathbb{X}) \geq a$. Indeed, by taking into account Remark 1(ii) and (iii), one finds that for every $x \in \mathbb{X}$ and $r>0$, for any $\epsilon>0$ it holds

$$
\begin{aligned}
\mathrm{B}(L(u), a r) & =\mathrm{B}(\Lambda u+C, a r)=\mathrm{B}(\Lambda u, a r)+C \\
& \subseteq \mathrm{B}(\Lambda x+C, r)+C=\mathrm{B}(\Lambda x+C+C, r)=\mathrm{B}(L(x)+C, r) .
\end{aligned}
$$

In the light of Example 3.2, if for instance $\Lambda \in \mathcal{L}\left(\mathbb{X}, \mathbb{R}^{m}\right)$ is such that sur $\Lambda>m$ and $C=\mathbb{R}_{+}^{m}$, then the resulting $L$ is metrically $\mathbb{R}_{+}^{m}$-increasing on $\mathbb{X}$ and $\operatorname{inc}(L ; \mathbb{X}) \geq \sqrt{m}$.

Example 3.4 (Locally regular nonlinear mapping). Recall that a mapping $f: X \longrightarrow$ $Y$ between metric spaces is said to be open at $\bar{x} \in X$ at a linear rate if there exist positive constants $\delta, \alpha$ and $\zeta$ such that

$$
f(\mathrm{~B}(x, r)) \supseteq \mathrm{B}(f(x), \alpha r) \cap \mathrm{B}(f(\bar{x}), \zeta), \quad \forall x \in \mathrm{B}(\bar{x}, \delta), \forall r \in(0, \delta) .
$$


Whenever $f$ is continuous at $\bar{x}$, inclusion (9) takes the simpler form

$$
f(\mathrm{~B}(x, r)) \supseteq \mathrm{B}(f(x), \alpha r), \quad \forall x \in \mathrm{B}(\bar{x}, \delta), \forall r \in(0, \delta) .
$$

The quantity

$$
\operatorname{sur}(f ; \bar{x})=\sup \{\alpha>0: \exists \delta>0 \text { such that inclusion (9) holds }\}
$$

is called the exact bound of local openness of $f$ at $\bar{x}$. Whenever $X$ and $Y$ are Banach spaces, according to the Lyusternik-Graves theorem, a mapping $f: \mathbb{X} \longrightarrow \mathbb{Y}$ strictly differentiable at $\bar{x} \in \mathbb{X}$ turns out to be open at a linear rate at $\bar{x}$ iff its strict derivative $\mathrm{D} f(\bar{x}) \in \mathcal{L}(\mathbb{X}, \mathbb{Y})$ is onto. Moreover, the following primal and dual estimates hold

$$
\operatorname{sur}(f ; \bar{x})=\frac{1}{\left\|\mathrm{D} f(\bar{x})^{-1}\right\|^{-}}=\inf _{\left\|u^{*}\right\|=1}\left\|\mathrm{D} f(\bar{x})^{\top} u^{*}\right\|
$$

(see, for instance, [6, Theorem 1.57]). Now, letting $\mathbb{Y}=\mathbb{R}^{m}, C=\mathbb{R}_{+}^{m}$ and $m \geq 2$, suppose that a mapping $f: \mathbb{X} \longrightarrow \mathbb{R}^{m}$ is continuous at $\bar{x}$ and such that

$$
\operatorname{sur}(f ; \bar{x})>m
$$

This means that there exists $\delta_{m}>0$ such that

$$
f(\mathrm{~B}(x, r)) \supseteq \mathrm{B}(f(x), m r), \quad \forall x \in \mathrm{B}\left(\bar{x}, \delta_{m}\right), \forall r \in\left(0, \delta_{m}\right) .
$$

Fix arbitrarily $x \in \mathrm{B}\left(\bar{x}, \delta_{m}\right)$ and $r \in\left(0, \delta_{m}\right)$, and define

$$
e_{r}=r \sqrt{m} \sum_{i=1}^{m} e^{i}
$$

As it is $\left\|e_{r}\right\|=r m$, one has $f(x)+e_{r} \in \mathrm{B}(f(x), m r)$. Thus, from inclusion (10), it follows that there exists $u \in \mathrm{B}(x, r)$ such that

$$
f(u)=f(x)+e_{r},
$$

while from the definition of $e_{r}$ it follows that $\mathrm{B}\left(e_{r}, r \sqrt{m}\right) \subseteq \mathbb{R}_{+}^{m}$. Consequently, one obtains

$$
\begin{aligned}
\mathrm{B}(f(u), r \sqrt{m}) & =\mathrm{B}\left(f(x)+e_{r}, r \sqrt{m}\right)=f(x)+\mathrm{B}\left(e_{r}, r \sqrt{m}\right) \subseteq f(x)+\mathbb{R}_{+}^{m} \\
& \subseteq \mathrm{B}\left(f(x)+\mathbb{R}_{+}^{m}, r\right) .
\end{aligned}
$$

This shows that, under the above assumptions, $f$ is metrically $\mathbb{R}_{+}^{m}$-increasing around $\bar{x}$, with $\operatorname{inc}(f ; \bar{x}) \geq \sqrt{m}$. By arguing as in Example 3.3, it is not difficult to see that the same property is shared with set-valued mappings of the form $f+\mathbb{R}_{+}^{m}$.

The next example exhibits a situation in which metric $C$-increase takes place in the absence of openness. 
Example 3.5. Let $X=\mathbb{Y}=\mathbb{R}$ be endowed with its usual Euclidean space structure, let $C=[0,+\infty)$ and let $f: \mathbb{R} \longrightarrow \mathbb{R}$ be given by

$$
f(x)=|x| .
$$

The mapping $f$ evidently fails to be open at a linear rate at $\bar{x}=0$. Nevertheless, as one easily checks, it turns out to be metrically $\mathbb{R}_{+}$-increasing on $\mathbb{R}$ with inc $(f ; \mathbb{R})=2$ (in particular, it is $\mathbb{R}_{+}$-increasing around $\bar{x}$ ).

A feature of the property under study to be pointed out is its robustness under additive Lipschitz continuous perturbations. Such a stability behaviour can be exploited to build further examples of metrically $C$-increasing mappings. In order to formulate this feature, let us recall that a set-valued mapping $G: X \rightrightarrows Y$ between metric spaces is said to be Lipschitz continuous on $X$ if there exists a constant $\beta \geq 0$ such that

$$
\operatorname{Haus}\left(G\left(x_{1}\right), G\left(x_{2}\right)\right) \leq \beta d\left(x_{1}, x_{2}\right), \quad \forall x_{1}, x_{2} \in X
$$

where Haus $\left(S_{1}, S_{2}\right)$ stands for the Hausdorff-Pompeiu distance of the sets $S_{1}$ and $S_{2}$, i.e.

$$
\operatorname{Haus}\left(S_{1}, S_{2}\right)=\max \left\{\operatorname{exc}\left(S_{1}, S_{2}\right), \operatorname{exc}\left(S_{2}, S_{1}\right)\right\}
$$

(on the Lipschitz properties of set-valued mappings see [5, Ch. 3C]). To avoid some major technicalities arising with translations of set enlargements in abstract spaces, the statement below is given for mappings taking values in a finite-dimensional Euclidean space.

Proposition 3.6. Let $F: X \rightrightarrows \mathbb{R}^{m}$ and $G: X \rightrightarrows \mathbb{R}^{m}$ be set-valued mappings and let $C \subset \mathbb{R}^{m}$ be a closed, convex cone. Suppose that:

(i) $F$ is metrically $C$-increasing on $X$;

(ii) $F+G: X \rightrightarrows \mathbb{R}^{m}$ is closed-valued;

(iii) $G$ is Lipschitz continuous on $X$ with a constant $\beta$ satisfying the condition

$$
\beta<1-\frac{1}{\operatorname{inc}(F ; X)}
$$

Then, the mapping $F+G: X \rightrightarrows \mathbb{R}^{m}$ is metrically $C$-increasing on $X$, with

$$
\operatorname{inc}(F+G ; X) \geq(1-\beta) \operatorname{inc}(F ; X) \text {. }
$$

Proof. Notice that hypothesis (iii) implies, in particular, the fact that $\beta<1$. Fix $x \in X$ and $r>0$. By virtue of the condition on $\beta$ in hypothesis (iii), it is possible to pick $a \in(1, \operatorname{inc}(F ; X))$ in such a way that

$$
a(1-\beta)>1 .
$$

Since $F$ is metrically $C$-increasing on $X$ with exact bound $\operatorname{inc}(F ; X)$, corresponding to $x$ and $(1-\beta) r>0$ there exists $u \in \mathrm{B}(x, r)$ such that

$$
\mathrm{B}(F(u), a(1-\beta) r) \subseteq \mathrm{B}(F(x)+C,(1-\beta) r) .
$$


Since $G$ is Lipschitz continuous on $X$ with a constant $\beta$, one has in particular

$$
\operatorname{exc}(G(u)), G(x)) \leq \beta d(u, x) \leq \beta r
$$

which entails

$$
G(u) \subseteq \mathrm{B}(G(x), \beta r)
$$

By taking into account Remark1(iii) and (v), from inclusions (13) and (12) one obtains

$$
\begin{aligned}
\mathrm{B}(F(u)+G(u), a(1-\beta) r) & =\mathrm{B}(F(u), a(1-\beta) r)+G(u) \\
& \subseteq \mathrm{B}(F(x)+C,(1-\beta) r)+\mathrm{B}(G(x), \beta r) \\
& \subseteq \mathrm{B}(F(x)+G(x), r) .
\end{aligned}
$$

This shows that $F+G$ is metrically $C$-increasing on $X$ and that $\operatorname{inc}(F+G ; X) \geq$ $a(1-\beta)$. Since the latter remains true for every $a \in(1, \operatorname{inc}(F ; X))$ fulfilling the condition in (11), the validity of the estimate in the thesis is also proved.

Remark 3. (i) It is worth noting that the hypothesis (iii) in Proposition 3.6 can be weakened by replacing the Lipschitz continuity of $G$ with a more general property called Lipschitz $C$-continuity, that postulates the existence of $\beta \geq 0$ such that

$$
\max \left\{\operatorname{exc}\left(G\left(x_{1}\right), G\left(x_{2}\right)+C\right), \operatorname{exc}\left(G\left(x_{2}\right), G\left(x_{1}\right)+C\right)\right\} \leq \beta d\left(x_{1}, x_{2}\right), \quad \forall x_{1}, x_{2} \in X
$$

(ii) Proposition 3.6 can be easily reformulated for mappings metrically $C$-increasing around a given point. In such an event, the Lipschitz continuity of $G$ on $X$ can be replaced with its local counterpart around the reference point.

(iii) It is well known that a sufficient condition for the sum of two closed sets to be still closed is that one of them is compact. Therefore, hypothesis (ii) is satisfied provided that, for instance, $G$ is compact-valued.

To the aim of better understanding the idea behind the notion of metric $C$-increase, it is useful to consider how it behaves in the very special case of single-valued scalar functions, with the cone $C=(-\infty, 0]$. In this setting, in its local version, the metric $C$-increase of $\varphi: X \longrightarrow \mathbb{R}$ around $\bar{x}$ prescribes the existence of $a>1$ such that, if in particular $x=\bar{x}$, an element $u \in \mathrm{B}(\bar{x}, r)$ can be found such that

$$
[\varphi(u)-a r, \varphi(u)+a r] \subseteq \mathrm{B}(\varphi(\bar{x})+(-\infty, 0], r)=\mathrm{B}((-\infty, \varphi(\bar{x})], r)=(-\infty, \varphi(\bar{x})+r] .
$$

This implies that $u \in \mathrm{B}(\bar{x}, r)$ must be such that

$$
\varphi(u)+a r \leq \varphi(\bar{x})+r
$$

and therefore it must hold

$$
\inf _{x \in \mathrm{B}(\bar{x}, r)} \varphi(x) \leq \varphi(\bar{x})-(a-1) r .
$$

The condition expressed by inequality (14) appears in what in variational analysis is referred to as a decrease principle. Such kind of statements provides conditions, expressed in terms of generalized derivatives (even in a metric space setting), upon which 
inequality (14) holds true. It found various applications in the analysis of solvability and error bounds for inequalities defined by l.s.c. scalar functions (see, among others, [4, Theorem 3.6.2] and [13, Ch. 1.6]). For instance, any function $\delta_{\varphi}: X \longrightarrow[0,+\infty]$ such that for every $x \in X$ and $r, c>0$

$$
\inf _{z \in \mathrm{B}(x, r)} \delta_{\varphi}(z) \geq c \quad \Longrightarrow \quad \inf _{z \in \mathrm{B}(x, r)} \varphi(z) \leq \varphi(x)-c r
$$

is called decrease index for $\varphi$. The strong slope of a function and its various subdifferential representations in Banach space settings are well-known examples of decrease index. Thus, metric $C$-increase can be viewed as a condition, directly formulated on setvalued mappings, which leads to generalize a behaviour that can be obtained through decrease principles.

\section{Solvability and error bounds}

The main question of this paper, which stimulated the introduction of the notion of metric $C$-increase, is the solvability of generalized equation of the form (GE) and related error bounds. As a prolegomenon to the analysis of such a question, it is worth pointing out the following basic topological property of the solution set $\operatorname{Sol}(F, C)$, which can be obtained at once under mild assumptions on the problem data.

Proposition 4.1. With reference to a given (GE), suppose that $F$ that is l.s.c. on $X$. Then, $\mathcal{S}$ ol $(F, C)$ is a (possibly empty) closed set. If $F$ is l.s.c. in a neighbourhood of $\bar{x} \in \mathcal{S}$ ol $(F, C)$, then $\mathcal{S}$ ol $(F, C)$ is locally closed around $\bar{x}$, i.e. there exists $\delta>0$ such that $\operatorname{Sol}(F, C) \cap \mathrm{B}(\bar{x}, \delta)$ is closed.

Proof. Since the convex cone $C$ is closed, it suffices to observe that $\mathcal{S}$ ol $(F, C)$ can be characterized as a sublevel set of the function $\phi$ defined as in (3), namely

$$
\mathcal{S} o l(F, C)=\{x \in X: \phi(x) \leq 0\}
$$

and then to recall Lemma $2.3(\mathrm{i})$.

One is now in a position to establish the main findings of the paper.

Theorem 4.2 (Solvability and global error bound). Given a set-valued mapping $F$ : $X \rightrightarrows \mathbb{Y}$, suppose that:

(i) $(X, d)$ is metrically complete;

(ii) $F$ is l.s.c. on $X$;

(iii) $F$ is metrically $C$-increasing on $X$, with exact bound inc $(F ; X)$.

Then, $\operatorname{Sol}(F, C) \neq \varnothing$ and the following estimate holds

$$
\operatorname{dist}(x, \mathcal{S} o l(F, C)) \leq \frac{\operatorname{exc}(F(x), C)}{\operatorname{inc}(F ; X)-1}, \quad \forall x \in X
$$

Proof. Consider the functional $\phi: X \longrightarrow[0,+\infty)$ defined as in (3) and take an arbitrary $a \in(1, \operatorname{inc}(F ; X))$. Notice that, by virtue of the lower semicontinuity of $F$ on $X$, in the light of Lemma 2.3(i), the function $\phi$ is l.s.c. on $X$ and it is bounded from below by definition. Take an arbitrary $x_{0} \in X$. If it is $\phi\left(x_{0}\right)=0$, then, as $C$ is 
closed, one has $F\left(x_{0}\right) \subseteq C$, so all the assertions in the thesis trivially happen to hold true. Assume now that $\phi\left(x_{0}\right)>0$. Since it is

$$
\phi\left(x_{0}\right) \leq \inf _{x \in X} \phi(x)+\phi\left(x_{0}\right)
$$

and the metric space $(X, d)$ has been assumed to be complete, according to the Ekeland variational principle (see [14]) for every $\lambda>0$ there exists $x_{\lambda} \in X$ such that

$$
\begin{gathered}
\phi\left(x_{\lambda}\right) \leq \phi\left(x_{0}\right), \\
d\left(x_{\lambda}, x_{0}\right) \leq \lambda,
\end{gathered}
$$

and

$$
\phi\left(x_{\lambda}\right)<\phi(x)+\frac{\phi\left(x_{0}\right)}{\lambda} d\left(x, x_{\lambda}\right), \quad \forall x \in X \backslash\left\{x_{\lambda}\right\} .
$$

Let us show that, if choosing

$$
\lambda=\frac{\phi\left(x_{0}\right)}{a-1}
$$

the corresponding $x_{\lambda} \in X$ is a zero of $\phi$. Ab absurdo, suppose that $\phi\left(x_{\lambda}\right)>0$. By hypothesis (ii), corresponding to $r=\phi\left(x_{\lambda}\right)$ there exists $u \in \mathrm{B}\left(x_{\lambda}, \phi\left(x_{\lambda}\right)\right)$ with the property

$$
\mathrm{B}\left(F(u), a \phi\left(x_{\lambda}\right)\right) \subseteq \mathrm{B}\left(F\left(x_{\lambda}\right)+C, \phi\left(x_{\lambda}\right)\right) .
$$

Notice that it must be $u \neq x_{\lambda}$, because for positive $r=\phi\left(x_{\lambda}\right)$ the last inclusion fails to be true, as observed in Remark 2. By applying Lemma 2.2 and Remark 1(iv), one obtains

$$
\begin{aligned}
\phi(u) & =\operatorname{exc}(F(u), C)=\operatorname{exc}\left(\mathrm{B}\left(F(u), a \phi\left(x_{\lambda}\right)\right), C\right)-a \phi\left(x_{\lambda}\right) \\
& \leq \operatorname{exc}\left(\mathrm{B}\left(F\left(x_{\lambda}\right)+C, \phi\left(x_{\lambda}\right)\right), C\right)-a \phi\left(x_{\lambda}\right) \\
& =\operatorname{exc}\left(F\left(x_{\lambda}\right)+C, C\right)+\phi\left(x_{\lambda}\right)-a \phi\left(x_{\lambda}\right) \\
& =\operatorname{exc}\left(F\left(x_{\lambda}\right), C\right)+(1-a) \phi\left(x_{\lambda}\right)=(2-a) \phi\left(x_{\lambda}\right) .
\end{aligned}
$$

If $a>2$, the above relation already yields a contradiction. Otherwise, by plugging $u$ in inequality (16) (remember that $u \neq x_{\lambda}$ ), one finds

$$
\phi\left(x_{\lambda}\right)<\phi(u)+(a-1) d\left(u, x_{\lambda}\right) \leq \phi\left(x_{\lambda}\right),
$$

wherefrom contradiction arises. This fact shows that, for the value of $\lambda$ chosen as above, it must happen that $x_{\lambda} \in \operatorname{Sol}(F, C)$. Moreover, as a consequence of inequality (15), by recalling formula (17), one obtains

$$
\operatorname{dist}\left(x_{0}, \mathcal{S} o l(F, C)\right) \leq d\left(x_{0}, x_{\lambda}\right) \leq \frac{\operatorname{exc}\left(F\left(x_{0}\right), C\right)}{a-1} .
$$


By arbitrariness of $a \in(1, \operatorname{inc}(F ; X))$, the last estimate completes the proof.

A generalized equation of the form (GE) can be regarded as a special case of a more general problem, which was called set-inclusion: given two set-valued mappings $\Psi: X \rightrightarrows Y$ and $\Phi: X \rightrightarrows Y$ between metric spaces

$$
\text { find } x \in X \text { such that } \Phi(x) \subseteq \Psi(x) \text {. }
$$

A result concerning the solvability and error bounds for set-valued inclusion problems was obtained in [15, Theorem 3.3], which relies on the notion of set-covering mapping. Nonetheless, Theorem 4.2 can not be derived as a special case from the aforementioned result. Indeed, with the identification $\Phi=F$ and $\Psi \equiv C$, the set-covering assumption on $\Psi$ appearing in Theorem 3.3 will be never satisfied. Moreover, in that theorem $\Phi$ is supposed to be Lipschitz continuous on $X$ with bounded values, what is not required in Theorem 4.2. In fact, even though exploiting an analogous variational technique of proof, the approach here proposed to address solvability and error bounds for (GE) is based on a different behaviour of the mapping $F$ : the reader should notice that the notion of metric $C$-increase needs a partial order structure on the range space $\mathbb{Y}$, whereas set-covering is a purely metric property.

After the seminal paper [21], A.V. Arutyunov and his research group developed a theory about coincidence points for set-valued mappings, aimed at unifying the wellknown results by Banach-Caccioppoli and Milyutin, where the property of openness plays a crucial role. Recall that, according to [21, First Definition], a mapping $f$ : $X \longrightarrow Y$ between metric spaces is said to be open (at a linear rate) on $X$ with constant $\alpha>0$ if

$$
f(\mathrm{~B}(x, r)) \supseteq \mathrm{B}(f(x), \alpha r), \quad \forall r \geq 0, \forall x \in X .
$$

Within this theory it is possible to derive a solvability result, with a related error bound estimate, for the following special case of (GE):

$$
\text { find } x \in X \text { such that } f(x) \in C \text {, }
$$

where $f: X \longrightarrow \mathbb{Y}$ is a given single-valued mapping. More precisely, it is possible to prove what follows: suppose that

(i) $(X, d)$ is metrically complete;

(ii) $f$ is continuous on $X$;

(iii) $f$ is open (at a linear rate) on $X$ with constant $\alpha$;

then $\mathcal{S} o l(f, C) \neq \varnothing$ and

$$
\operatorname{dist}(x, \mathcal{S} o l(f, C)) \leq \frac{\operatorname{dist}(f(x), C)}{\alpha}, \quad \forall x \in X .
$$

Since, as mentioned above, the properties of metric $C$-increase and openness are independent, a single-valued specialization of Theorem 4.2 seems not be achievable within the theory developed after [21].

In optimization contexts, where local optimality is to be investigated, often local error bound estimates are required. Such a need motivates the interest in a local version of the previous result. Its proof is provided in detail inasmuch as the variational technique employed in Theorem 4.2 must be submitted to nontrivial adjustments, when referred to a local setting. 
Theorem 4.3 (Local error bound). Let $F: X \rightrightarrows \mathbb{Y}$ be a set-valued mapping and let $\bar{x} \in \mathcal{S}$ ol $(F, C)$. Suppose that:

(i) $(X, d)$ is metrically complete;

(ii) $F$ is l.s.c. in a neighbourhood of $\bar{x}$ and Hausdorff $C$-u.s.c. at $\bar{x}$;

(iii) $F$ is metrically $C$-increasing around $\bar{x}$ with exact bound $\operatorname{inc}(F ; \bar{x})$.

Then, for every $a \in(1, \operatorname{inc}(F ; \bar{x}))$ there exists $\delta_{a}>0$ such that

$$
\operatorname{dist}(x, \mathcal{S} o l(F, C)) \leq \frac{\operatorname{exc}(F(x), C)}{a-1}, \quad \forall x \in \mathrm{B}\left(\bar{x}, \delta_{a}\right)
$$

Proof. Fixed any $a \in(1, \operatorname{inc}(F ; \bar{x}))$, by hypothesis (iii) there exists $\delta_{0}>0$ such that

$$
\forall x \in \mathrm{B}\left(\bar{x}, \delta_{0}\right), \forall r \in\left(0, \delta_{0}\right), \exists u \in \mathrm{B}(x, r): \mathrm{B}(F(u), a r) \subseteq \mathrm{B}(F(x)+C, r) .
$$

Consider once again the function $\phi$ defined as in (3). By virtue of the lower semicontinuity of $F$ in a neighbourhood of $\bar{x}$, in force of Lemma 2.3(i), there exists $\delta_{1}>0$ such that $\phi$ is l.s.c. at each point of $\mathrm{B}\left(\bar{x}, \delta_{1}\right)$. Furthermore, as $F$ is also Hausdorff $C$-u.s.c. at $\bar{x}, \phi$ turns out to be, in particular, continuous at $\bar{x}$. Since $\phi(\bar{x})=0$ because $\bar{x} \in \mathcal{S}$ ol $(F, C)$, corresponding to $\delta_{0}$ there exists $\delta_{2}>0$ such that

$$
\phi(x) \leq \frac{\delta_{0}}{2}, \quad \forall x \in \mathrm{B}\left(\bar{x}, \delta_{2}\right) .
$$

Now, let us define

$$
\delta_{*}=\min \left\{\delta_{0}, \delta_{1}, \delta_{2}\right\}
$$

and set $\delta_{a}=\delta_{*} / 4$

To show the validity of the inequality in the thesis, fix an arbitrary $x_{0} \in$ $\mathrm{B}\left(\bar{x}, \delta_{a}\right) \backslash \mathcal{S} o l(F, C)$. The fact that $x_{0} \notin \mathcal{S}$ ol $(F, C)$ implies $\phi\left(x_{0}\right)>0$. Consider the function $\phi: \mathrm{B}\left(x_{0}, \delta_{a}\right) \longrightarrow[0,+\infty)$. Observe that, as it is true that $\mathrm{B}\left(x_{0}, \delta_{a}\right) \subseteq \mathrm{B}\left(\bar{x}, \delta_{*} / 2\right) \subseteq$ $\mathrm{B}\left(\bar{x}, \delta_{1}\right), \phi$ is l.s.c. on $\mathrm{B}\left(x_{0}, \delta_{a}\right)$. This set, as a closed subset of a complete metric space, is complete. Therefore it is possible to apply the Ekeland variational principle. It ensures that, corresponding to $\lambda=\frac{\phi\left(x_{0}\right)}{a-1}$, there exists $x_{\lambda} \in \mathrm{B}\left(x_{0}, \delta_{a}\right)$ such that

$$
\begin{gathered}
\phi\left(x_{\lambda}\right) \leq \phi\left(x_{0}\right)-(a-1) d\left(x_{\lambda}, x_{0}\right), \\
d\left(x_{\lambda}, x_{0}\right) \leq \frac{\phi\left(x_{0}\right)}{a-1}
\end{gathered}
$$

and

$$
\phi\left(x_{\lambda}\right)<\phi(x)+(a-1) d\left(x, x_{\lambda}\right), \quad \forall x \in \mathrm{B}\left(x_{0}, \delta_{a}\right) \backslash\left\{x_{\lambda}\right\} .
$$

If $\phi\left(x_{\lambda}\right)=0$ one gets $x_{\lambda} \in \mathcal{S}$ ol $(F, C)$ and hence, by taking into account inequality (22) the thesis is proved. Suppose that $\phi\left(x_{\lambda}\right)>0$. In such an event, by inequality (21) one has $\phi\left(x_{\lambda}\right) \leq \phi\left(x_{0}\right)$ so, from the fact that $x_{0} \in \mathrm{B}\left(\bar{x}, \delta_{a}\right) \subseteq \mathrm{B}\left(\bar{x}, \delta_{2}\right)$ one obtains

$$
\phi\left(x_{\lambda}\right) \leq \phi\left(x_{0}\right) \leq \frac{\delta_{0}}{2}
$$


On the other hand, since it holds

$$
d\left(x_{\lambda}, \bar{x}\right) \leq d\left(x_{\lambda}, x_{0}\right)+d\left(x_{0}, \bar{x}\right) \leq \frac{\delta_{*}}{2}<\delta_{0},
$$

one has $x_{\lambda} \in \mathrm{B}\left(\bar{x}, \delta_{0}\right)$. The last two facts make it possible to apply inclusion (19), with $x=x_{\lambda}$ and $r=\phi\left(x_{\lambda}\right)$, so that one gets the existence of $u \in \mathrm{B}\left(x_{\lambda}, \phi\left(x_{\lambda}\right)\right)$ such that

$$
\mathrm{B}\left(F(u), a \phi\left(x_{\lambda}\right)\right) \subseteq \mathrm{B}\left(F\left(x_{\lambda}\right)+C, \phi\left(x_{\lambda}\right)\right)
$$

Now notice that, whenever it happens that $a>2$, then by arguing in the same way as in the proof of Theorem 4.2, one readily reaches a contradiction. Thus, it is possible to assume henceforth that $a \in(1,2]$. If it happens that $d\left(u, x_{0}\right) \leq \delta_{a}$, then inequality (23) can be exploited. Thus, by plugging $u$ in this inequality and proceeding exactly as done for the proof of Theorem 4.2, one reaches a contradiction, thereby proving that it must be $\phi\left(x_{\lambda}\right)=0$. So, in the current case, the thesis is proved. Otherwise, if it happens that $d\left(u, x_{0}\right)>\delta_{a}=\delta_{*} / 4$, it is useful to observe that, on the account of inequalities (21) and (22), it holds

$$
\begin{aligned}
d\left(u, x_{0}\right) & \leq d\left(u, x_{\lambda}\right)+d\left(x_{\lambda}, x_{0}\right) \leq \phi\left(x_{0}\right)-(a-1) d\left(x_{\lambda}, x_{0}\right)+d\left(x_{\lambda}, x_{0}\right) \\
& =\phi\left(x_{0}\right)-(a-2) d\left(x_{\lambda}, x_{0}\right) \leq \phi\left(x_{0}\right)-\frac{a-2}{a-1} \phi\left(x_{0}\right)=\frac{\phi\left(x_{0}\right)}{a-1}
\end{aligned}
$$

Consequently, by recalling that $\bar{x} \in \mathcal{S}$ ol $(F, C)$, one finds

$$
\operatorname{dist}\left(x_{0}, \mathcal{S o l}(F, C)\right) \leq d\left(x_{0}, \bar{x}\right) \leq \frac{\delta_{*}}{4} \leq \frac{\phi\left(x_{0}\right)}{a-1} .
$$

The last inequality, by arbitrariness of $x_{0}$, completes the proof.

\section{Applications to optimization}

\subsection{Existence of ideal efficient solutions}

Let us consider a vector optimization problem (VOP). As a straightforward consequence of Theorem 4.2, the following result concerning the existence of ideal efficient solutions to (VOP) and the distance from ideal efficiency can be established. In its statement, by $\operatorname{IE}(f ; R)$ the set of all ideal $C$-efficient solutions to (VOP) is denoted. In view of the employment of Proposition 3.6, the range space $\mathbb{Y}$ is supposed to be a finite-dimensional Euclidean space, partially ordered by a closed, convex cone $C$.

Theorem 5.1. With reference to a vector optimization problem (VOP), suppose that:

(i) $(X, d)$ is metrically complete;

(ii) $f: X \longrightarrow \mathbb{R}^{m}$ is continuous on $R$;

(iii) $-f$ is metrically $C$-increasing on $R$;

(iv) sets $R$ and $f(R)$ are closed.

Then, the set $\operatorname{IE}(f ; R)$ is nonempty and closed and it holds

$$
\operatorname{dist}(x, \operatorname{IE}(f ; R)) \leq \frac{\operatorname{exc}(f(R)-f(x), C)}{\operatorname{inc}(-f ; R)-1}, \quad \forall x \in R .
$$


Proof. As pointed out in Example 1.1(i), IE $(f ; R)$ coincides with $\mathcal{S}$ ol $(f(R)-f, C)$. In order to apply Theorem 4.2, observe that, by virtue of hypotheses (ii) and (iv), the set-valued mapping $F: R \rightrightarrows \mathbb{R}^{m}$, defined by $F(x)=f(R)-f(x)$, is closed-valued and l.s.c. on $R$. This fact enables one to invoke Proposition 4.1, which says that $\operatorname{IE}(f ; R)$ is closed. Since $-f$ is metrically $C$-increasing on $R$ and the mapping $G: R \rightrightarrows \mathbb{R}^{m}$, given by $G \equiv f(R)$ is evidently Lipschitz continuous with constant $\beta=0$, Proposition 3.6 ensures that $F$, as an additive perturbation of $-f$, is still metrically $C$-increasing on $R$, with

$$
\operatorname{inc}(F ; R) \geq \operatorname{inc}(-f ; R) \text {. }
$$

As a closed subset of a complete metric space, $(R, d)$ is a complete metric space. Then, it is possible to apply Theorem 4.2, from which all the remaining assertions in the thesis follow.

As a comment to Theorem 5.1, let us remark that, in contrast with the most part of existence results in vector optimization (see [7,16]), no kind of compactness is assumed on $f(R)$. The existence of ideal efficient solutions comes as a consequence of the interplay between the metric behaviour of $f$ and the partial order $C$, and metric completeness.

\subsection{Penalization in constrained optimization}

This subsection deals with constrained (scalar) optimization problems, whose constraint system is defined by a generalized equation of the form (GE), namely

$$
\min \varphi(x) \quad \text { subject to } \quad F(x) \subseteq C \text {. }
$$

The objective function $\varphi: X \longrightarrow \mathbb{R}$ is assumed to be locally Lipschitz at $\bar{x}$, i.e. with the property that there exist $\beta \geq 0$ and $\delta>0$ such that

$$
\left|\varphi\left(x_{1}\right)-\varphi\left(x_{2}\right)\right| \leq \beta d\left(x_{1}, x_{2}\right), \quad \forall x \in \mathrm{B}(\bar{x}, \delta) .
$$

The exact local Lipschitz bound of $\varphi$ at $\bar{x}$ is denoted in what follows by

$$
\operatorname{lip}(\varphi ; \bar{x})=\inf \{\beta \geq 0: \exists \delta>0 \text { such that inclusion (24) holds }\}
$$

Let us indicate by $\mathcal{R}$ the feasible region of $(\mathcal{P})$, that is $\mathcal{R}=\operatorname{Sol}((F, C))$.

According to a widely-exploited scheme of analysis (see, for instance, [17, Ch. 6]), whenever an error bound for a given constraint system is established, a corresponding exact penalization result is derived in a standard way. It is worth noting that penalty method is an approach developed within constrained optimization after [18,19], which consists in reducing a given constrained optimization problem to an unconstrained one, by replacing its objective function with a so-called penalty functional. In the case of problem $(\mathcal{P})$, the penalty functional is defined as

$$
\varphi_{\lambda}(x)=\varphi(x)+\lambda \operatorname{exc}(F(x), C)
$$

where $\lambda$ is a positive parameter and the additional term clearly quantifies the constraint violation. In this context, a penalty function $\varphi_{\lambda}$ is said to be exact at a local 
solution $\bar{x} \in \mathcal{R}$ to $(\mathcal{P})$ provided that $\bar{x}$ is also a local solution to the following unconstrained problem

$$
\min _{x \in X} \varphi_{\lambda}(x)
$$

The next result provides a sufficient condition for the exactness of a penalty function, along with a quantitative estimate, expressed in term of problem data, for a penalty parameter $\lambda$ to given an exact penalty function.

Theorem 5.2 (Exact penalization). Given a constrained optimization problem $(\mathcal{P})$, suppose that:

(i) $(X, d)$ is metrically complete;

(ii) $\bar{x} \in \mathcal{R}$ is a local solution to $(\mathcal{P})$;

(iii) $\varphi$ is locally Lipschitz at $\bar{x}$;

(iv) $F$ is l.s.c. in a neighbourhood of $\bar{x}$ and Hausdorff C-u.s.c. at $\bar{x}$;

(v) $F$ is metrically $C$-increasing around $\bar{x}$.

Then, for every $\lambda>\frac{\operatorname{lip}(\varphi ; \bar{x})}{\operatorname{inc}(F ; \bar{x})-1}$, the function $\varphi_{\lambda}$ is exact at $\bar{x}$.

Proof. Fix $\lambda>\frac{\operatorname{lip}(\varphi ; \bar{x})}{\operatorname{inc}(F ; \bar{x})-1}$. Then, it is possible to pick constants $a, \tilde{a}$ and $\beta$, with

$$
\beta>\operatorname{lip}(\varphi ; \bar{x}) \quad \text { and } \quad 1<\tilde{a}<a<\operatorname{inc}(F ; \bar{x})
$$

in such a way that

$$
\lambda>\frac{\beta}{\tilde{a}-1}>\frac{\beta}{a-1}>\frac{\operatorname{lip}(\varphi ; \bar{x})}{\operatorname{inc}(F ; \bar{x})-1} .
$$

As a consequence of hypothesis (ii), there exists $\delta_{0}$ such that

$$
\varphi(x) \geq \varphi(\bar{x}), \quad \forall x \in \mathrm{B}\left(\bar{x}, \delta_{0}\right) \cap \mathcal{R} .
$$

By virtue of hypothesis (iii), there exists $\delta_{\beta}>0$ such that

$$
\left|\varphi\left(x_{1}\right)-\varphi\left(x_{2}\right)\right| \leq \beta d\left(x_{1}, x_{2}\right), \quad \forall x \in \mathrm{B}\left(\bar{x}, \delta_{\beta}\right) .
$$

Since, under the hypotheses (i), (iv) and (iv) it is possible to apply Theorem 4.3, there exists $\delta_{a}>0$ such that

$$
\operatorname{dist}(x, \mathcal{R}) \leq \frac{\operatorname{exc}(F(x), C)}{a-1}, \quad \forall x \in \mathrm{B}\left(\bar{x}, \delta_{a}\right)
$$

Now, define

$$
r_{*}=\frac{1}{4} \min \left\{\delta_{0}, \delta_{\beta}, \delta_{a}\right\} .
$$

According to the definition of $\varphi_{\lambda}$, clearly it is

$$
\varphi_{\lambda}(x)=\varphi(x) \geq \varphi(\bar{x})=\varphi_{\lambda}(\bar{x}), \quad \forall x \in \mathrm{B}\left(\bar{x}, r_{*}\right) \cap \mathcal{R} .
$$


It remains to prove that $\varphi_{\lambda}$ fulfils a similar inequality also for every $x \in \mathrm{B}\left(\bar{x}, r_{*}\right) \backslash \mathcal{R}$. To this aim, fix an arbitrary $x \in \mathrm{B}\left(\bar{x}, r_{*}\right) \backslash \mathcal{R}$. Since $r_{*}<\delta_{a}$, inequality (28) is valid at each point of $\mathrm{B}\left(\bar{x}, r_{*}\right)$ and hence

$$
\operatorname{dist}(x, \mathcal{R})<\frac{\operatorname{exc}(F(x), C)}{\tilde{a}-1} .
$$

Remember that, as $x \notin \mathcal{R}$, it must be $\operatorname{exc}(F(x), C)>0)$. Moreover, under the aforementioned hypothesis of lower semicontinuity on $F$, in the light of Proposition $4.1 \mathcal{R}$ turns out to be locally closed around $\bar{x}$ and hence one has also dist $(x, \mathcal{R})>0$. These facts imply the existence of $z \in \mathcal{R}$ such that

$$
d(x, z)<2 \operatorname{dist}(x, \mathcal{R})
$$

and

$$
d(x, z)<\frac{\operatorname{exc}(F(x), C)}{\tilde{a}-1} .
$$

Notice that, since it is $\bar{x} \in \mathcal{R}$, then by inequality (30) one gets

$$
d(x, z)<2 d(x, \bar{x}) \leq 2 r_{*} .
$$

Owing to the definition of $r_{*}$ made in (29), it follows

$$
d(z, \bar{x}) \leq d(z, x)+d(x, \bar{x}) \leq 3 r_{*}<\min \left\{\delta_{0}, \delta_{\beta}, \delta_{a}\right\} .
$$

As a consequence, one obtains

$$
z \in \mathcal{R} \cap \mathrm{B}\left(\bar{x}, \delta_{0}\right) \cap \mathrm{B}\left(\bar{x}, \delta_{\beta}\right)
$$

This fact enables one to apply inequalities (26) and (27). Thus, by recalling inequality (31), one finds

$$
\varphi(x) \geq \varphi(z)-\beta d(x, z) \geq \varphi(\bar{x})-\beta \frac{\operatorname{exc}(F(x), C)}{\tilde{a}-1}
$$

wherefrom, in the light of inequality (25), one deduces

$$
\varphi_{\lambda}(x) \geq \varphi(\bar{x})=\varphi_{\lambda}(\bar{x}) .
$$

The last inequality completes the proof.

Theorem 5.2 can be used as a starting point for deriving necessary optimality conditions, when problem $(\mathcal{P})$ is considered in more structured settings. For instance, if $F: \mathbb{X} \rightrightarrows \mathbb{Y}$ is a set-valued mapping between normed spaces with the property

$$
F\left(t x_{1}+(1-t) x_{2}\right) \subseteq t F\left(x_{1}\right)+(1-t) F\left(x_{2}\right), \quad \forall x_{1}, x_{2} \in \mathbb{X}, \forall t \in[0,1]
$$

(this happens, for example, whenever $F$ is defined by a set of linear operators), then the function $x \mapsto \operatorname{exc}(F(x), C)$ turns out to be convex. Thus, under the assumptions 
of Theorem 5.2, a necessary optimality condition can be readily expressed in terms of the Clarke subdifferential (see [20, Ch. 10]). In other cases, to derive a verifiable necessary optimality condition, it may be useful to handle the term $x \mapsto \operatorname{exc}(F(x), C)$ by other kinds of subdifferential (sometimes more elaborated), which are available in nonsmooth analysis (see [4, 6, 9, 13]). It is worth mentioning that some of them may perform better than the Clarke subdifferential, even in the Lipschitz case, inasmuch as they lead to smaller constructions in the dual space.

Results of exact penalization are often complemented with conditions upon which from the global strict optimality of an element for a penalized problem one gets its (global) optimality for the original problem. A similar result can be achieved also in the context of problem $(\mathcal{P})$, by employing this time the global error bound estimate for generalized equations (GE).

Proposition 5.3. With reference to a problem $(\mathcal{P})$, suppose that:

(i) $(X, d)$ is metrically complete;

(ii) $(\mathcal{P})$ admits global solutions;

(iii) $\varphi$ is Lipschitz continuous on $X$;

(iv) $F$ is l.s.c. on $X$;

(v) $F$ is metrically $C$-increasing on $X$.

If $\hat{x} \in X$ is a strict global solution to problem $\left(\mathcal{P}_{\lambda_{\epsilon}}\right)$, with

$$
\lambda_{\epsilon}=\frac{(1+\epsilon) \beta}{\operatorname{inc}(F ; X)-1}
$$

for some $\epsilon>0$, then $\hat{x}$ is a global solution also to $(\mathcal{P})$.

Proof. Once proved that $\hat{x} \in \mathcal{R}$ the thesis follows at once, so assume, ab absurdo, that $\hat{x} \notin \mathcal{R}$. Under the above hypotheses it is possible to apply Theorem 4.2 and, in particular, the error bound estimate in its thesis. This amounts to say that, corresponding to $\epsilon>0$, there exists $x_{\epsilon} \in \mathcal{R}$ such that

$$
d\left(x_{\epsilon}, \hat{x}\right) \leq \frac{(1+\epsilon) \operatorname{exc}(F(\hat{x}), C)}{\operatorname{inc}(F ; X)-1} .
$$

According to hypothesis (ii), let $\bar{x} \in \mathcal{R}$ be a global solution to $(\mathcal{P})$. From the global optimality of $\hat{x}$ for $\left(\mathcal{P}_{\lambda_{\epsilon}}\right)$, inequality (32) and hypthesis (iii), it follows

$$
\begin{aligned}
\varphi(\bar{x}) & =\varphi_{\lambda_{\epsilon}}(\bar{x}) \geq \varphi_{\lambda_{\epsilon}}(\hat{x})=\varphi(\hat{x})+\lambda_{\epsilon} \operatorname{exc}(F(\hat{x}), C) \\
& \geq \varphi\left(x_{\epsilon}\right)-\beta d\left(\hat{x}, x_{\epsilon}\right)+\lambda_{\epsilon} \operatorname{exc}(F(\hat{x}), C) \geq \varphi\left(x_{\epsilon}\right) \\
& \geq \varphi(\bar{x}) .
\end{aligned}
$$

The above inequalities imply that $\varphi_{\lambda_{\epsilon}}(\hat{x})=\varphi_{\lambda_{\epsilon}}\left(x_{\epsilon}\right)$. Since $\hat{x}$ has been supposed to be a strict global solution to $\left(\mathcal{P}_{\lambda_{\epsilon}}\right)$, the last equality forces $\hat{x}=x_{\epsilon}$, but this contradicts the fact that $\hat{x} \notin \mathcal{R}$. The proof is complete.

Acknowledgements The author thanks the anonymous referees for valuable comments. 


\section{References}

[1] Robinson SM. Generalized equations and their solutions, Part I: Basic theory. Point-to-set maps and mathematical programming. Math. Programming Stud. 1979; 10: 128-141.

[2] Robinson SM. Strongly regular generalized equations. Math. Oper. Res. 1980; 5: 43-62.

[3] Bonnans JF, Shapiro A. Perturbation analysis of optimization problems. Springer Series in Operations Research. New York: Springer-Verlag; 2000.

[4] Borwein JM, Zhu QJ. Techniques of variational analysis. New York: Springer-Verlag; 2005.

[5] Dontchev AL, Rockafellar RT. Implicit functions and solution mappings. A view from variational analysis. 2nd ed. New York: Springer; 2014.

[6] Mordukhovich BS. Variational analysis and generalized differentiation. I. Basic theory. Berlin: Springer-Verlag; 2006.

[7] Jahan J. Vector optimization. Theory, applications, and extensions. Berlin: SpringerVerlag; 2004.

[8] Hettich R, Kortanek KO. Semi-infinite programming: Theory, methods, and applications. SIAM Review 1993; 35(3): 380-429.

[9] Schirotzek W. Nonsmooth Analysis. Berlin: Springer; 2007.

[10] Färe R. Fundamentals of production theory. Berlin: Springer-Verlag; 1988.

[11] Rasmussen S. Production economics. The basic theory of production optimisation. 2nd ed. Heidelberg: Springer; 2013.

[12] Castellani M. Error bounds for set-valued maps. Generalized convexity and optimization for economic and financial decisions. Bologna: Pitagora; 1998; 121-135.

[13] Penot J-P. Calculus without derivative. New York: Springer; 2013.

[14] Ekeland I. On the variational principle. J. Math. Anal. Appl. 1974; 47: 324-353.

[15] Uderzo A. On a set-covering property of multivalued mappings. Pure Appl. Funct. Anal. 2017; 2(1): 129-151.

[16] Luc DT. Theory of vector optimization. Berlin: Springer-Verlag; 1989.

[17] Facchinei F, Pang J-S. Finite-dimensional variational inequalities and complementarity problems. Vol. I. New York: Springer-Verlag; 2003.

[18] Eremin I.I. The method of penalties in convex programming, Dokl. Akad. Nauk SSSR 1967; 173: 748-751.

[19] Zangwill W.I. Nonlinear programming via penalty functions Management Science 1967; 13: $344-358$.

[20] Clarke F. Functional analysis, calculus of variations and optimal control. London: Springer; 2013.

[21] Arutyunov, AV. Covering mappings in metric spaces, and fixed points. Dokl. Math. 2007; 76(2): 665-668. 